\title{
SOLUSI SISTEM PERSAMAAN DIFERENSIAL FRACTIONAL LINIER POSITIF
}

\author{
KHOLIJAH LUBIS, ZULAKMAL \\ Program Studi S1 Matematika, \\ Fakultas Matematika dan Ilmu Pengetahuan Alam, Universitas Andalas, \\ Kampus UNAND Limau Manis Padang, Indonesia. \\ email : kholijahlubis1995@gmail.com
}

Diterima 9 Maret 2019 Direvisi 7 April 2019 Dipublikasikan 7 Mei 2019

\begin{abstract}
Abstrak. Dalam makalah ini dikaji solusi dari sistem persamaan diferensial fractional linier. Solusi sistem persamaan diferensial fractional linier diperoleh dengan menerapkan transformasi Laplace. Sistem persamaan diferensial linier fractional dikatakan positif jika untuk setiap keadaan awal non- negatif, maka trajektori dari sistem linier tersebut adalah non-negatif dengan berlalunya waktu.

Kata Kunci: Sistem Fractional, Sistem Fractional positif, Transformasi Laplace.
\end{abstract}

\section{Pendahuluan}

Diberikan suatu sistem persamaan diferensial Fractional linier sebagai berikut :

dengan

$$
\frac{d^{\alpha} \mathbf{x}(t)}{d t^{\alpha}}=A \mathbf{x}(t)+B \mathbf{u}(t), \quad \mathbf{x}(0)=\mathbf{x}_{0}
$$

$$
\frac{d^{\alpha} f(t)}{d t^{\alpha}}=\frac{1}{\Gamma(k-\alpha)} \int_{0}^{t} \frac{f^{(k)}(\tau) d \tau}{(t-\tau)^{\alpha+1-k}}
$$

untuk $k-1<\alpha<k, k \in \mathbb{N}$, dan $\Gamma$ adalah fungsi Gamma, dimana $A \in \mathbb{R}^{n \times n}$, $\mathbf{x} \in \mathbb{R}^{n}$ dan $t \in \mathbb{R}_{+}$.

Sistem (1.1) dikatakan positif jika untuk setiap keadaan awal $\mathbf{x}_{0} \in \overline{\mathbb{R}}_{+}^{n}$ dan $t \geq 0$ maka solusi dari (1.1) adalah non-negatif, yaitu $\mathbf{x}(t) \in \overline{\mathbb{R}}_{+}^{n}$. Kajian dari sistem linier dimulai dengan menerapkan transformasi Laplace pada Sistem (1.1). Dalam makalah ini dikaji solusi dari sistem persamaan diferensial fractional linier dengan asumsi bahwa sistem (1.1) adalah positif dengan menerapkan transformasi Laplace.

\section{Tinjauan Pustaka}

Definisi 2.1. [6] Matriks $A=\left[a_{i j}\right] \in \mathbb{R}^{n \times n}$ dikatakan matriks Metzler jika entrientri selain entri diagonal utamanya adalah non-negatif, yaitu $a_{i j} \geq 0$ untuk $i \neq j$, $i, j=1,2, \cdots, n$. 
Definisi 2.2. [3] Misalkan $\mathbf{f}(t) \in \mathbb{R}^{n}$ adalah suatu fungsi vektor yang berada dalam interval $[0,+\infty)$. Transformasi Laplace dari fungsi $\mathbf{f}(t)$ didefinisikan sebagai berikut

$$
\mathcal{L}[\mathbf{f}(t)]=\mathbf{F}(s)=\int_{0}^{\infty} \mathbf{f}(t) e^{-s t} d t
$$

$\operatorname{asalkan} \int_{0}^{\infty} f_{i}(t) e^{-s t} d t$ ada, untuk $i=1,2, \cdots, n$.

Teorema 2.3. [6] Jika transformasi Laplace dari fungsi $\mathbf{f}(t)$ dan $g(t)$ adalah $\mathbf{F}(s)$ dan $G(s)$ maka

$$
\mathcal{L}\left[\int_{0}^{t} \mathbf{f}(t-\tau) g(\tau) d \tau\right]=\mathbf{F}(s) \backslash G(s) .
$$

Definisi 2.4. [6] Fungsi Gamma yang dinotasikan dengan $\Gamma(x)$ didefinisikan sebagai berikut

$$
\Gamma(x)=\int_{0}^{\infty} t^{x-1} e^{-t} d t, x>0 .
$$

Definisi 2.5. [6] Turunan fractional Caputo orde $\alpha \in \mathbb{R}$ dari fungsi $f(t)$, dinotasikan dengan $\frac{d^{\alpha}}{d t^{\alpha}}$, didefinisikan sebagai berikut :

$$
\frac{d^{\alpha} f(t)}{d t^{\alpha}}=\frac{1}{\Gamma(k-\alpha)} \int_{0}^{t} \frac{f^{(k)}(\tau) d \tau}{(t-\tau)^{\alpha+1-k}} \text { dengan } k-1<\alpha<k, k \in \mathbb{N} .
$$

Dari Definisi 2.5 dapat diperoleh hasil transformasi Laplace dari turunan fractional Caputo dengan orde $\alpha \in \mathbb{R}$ yang disajikan dalam teorema di bawah ini .

Teorema 2.6. [6] Transformasi Laplace dari turunan fractional Caputo adalah

$$
\mathcal{L}\left[\frac{d^{\alpha}}{d t^{\alpha}} f(t)\right]=s^{\alpha} F(s)-\sum_{k=1}^{m} s^{(\alpha-k)} f^{(k-1)}\left(0^{+}\right) .
$$

Bukti.

$$
\begin{aligned}
\mathcal{L}\left[\frac{d^{\alpha}}{d t^{\alpha}} f(t)\right] & =\mathcal{L}\left[\frac{1}{\Gamma(m-\alpha)} \int_{0}^{t} \frac{f^{(m)}(\tau) d \tau}{(t-\tau)^{\alpha+1-m}}\right] \\
& =\frac{1}{\Gamma(m-\alpha)} \mathcal{L}\left[\int_{0}^{t} \frac{f^{(m)}(\tau) d \tau}{(t-\tau)^{\alpha+1-m}}\right]
\end{aligned}
$$

Berdasarkan Teorema 2.3 maka

$$
\begin{aligned}
& =\frac{1}{\Gamma(m-\alpha)} \frac{\Gamma(m-\alpha)}{s^{m-\alpha}}\left[s^{m} F(s)-\sum_{k=1}^{m} s^{(m-k)} f^{(k-1)}(0)\right] \\
& =s^{\alpha} F(s)-\sum_{k=1}^{m} s^{(\alpha-k)} f^{(k-1)}(0) .
\end{aligned}
$$

\section{Pembahasan}

Matriks $A=\left[a_{i j}\right] \in \mathbb{R}^{n \times n}$ dikatakan matriks Metzler jika entri-entri selain entri diagonal utamanya adalah non-negatif, yaitu $a_{i j} \geq 0$ untuk $i \neq j, i, j=1,2, \cdots, n$ 


\subsection{Sistem Linier Fractional}

Perhatikan kembali sistem (1.1). Dengan menerapkan transformasi Laplace pada sistem (1.1), diperoleh

$$
\mathcal{L}\left[\frac{d^{\alpha}}{d t^{\alpha}} \mathbf{x}(t)\right]=\mathcal{L}[A \mathbf{x}(t)+B \mathbf{u}(t)], \text { dengan } 0<\alpha<1
$$

Berdasarkan Teorema 2.3 dan Teorema 2.6, diperoleh

$$
\left[I_{n} s^{\alpha}-A\right] \mathbf{X}(s)=s^{\alpha-1} \mathbf{x}_{0}+B \mathbf{U}(s)
$$

Jika $\operatorname{det}\left[I_{n} s^{\alpha}-A\right] \neq 0$ maka persamaan (3.1) menjadi

$$
\begin{gathered}
\mathbf{X}(s)=\left[I_{n} s^{\alpha}-A\right]^{-1}\left[s^{\alpha-1} \mathbf{x}_{0}+B \mathbf{U}(s)\right] . \\
{\left[I_{n} s^{\alpha}-A\right]^{-1}=\sum_{k=0}^{\infty} A^{k} s^{-(k+1) \alpha}}
\end{gathered}
$$

Dengan mensubtitusikan persamaan (3.3) ke dalam persamaan (3.2), diperoleh

$$
\mathbf{X}(s)=\sum_{k=0}^{\infty} A^{k} s^{-(k \alpha+1)} \mathbf{x}_{0}+\sum_{k=0}^{\infty} A^{k} s^{-(k+1) \alpha} B \mathbf{U}(s)
$$

Invers transformasi Laplace dari persamaan (3.4) adalah

$$
\begin{aligned}
\mathbf{x}(t) & =\mathcal{L}^{-1}[\mathbf{X}(s)] \\
& =\mathcal{L}^{-1}\left[\sum_{k=0}^{\infty} A^{k} s^{-(k \alpha+1)} \mathbf{x}_{0}+\sum_{k=0}^{\infty} A^{k} s^{-(k+1) \alpha} B \mathbf{U}(s)\right] \\
& =\sum_{k=0}^{\infty} A^{k} \mathcal{L}^{-1}\left[s^{-(k \alpha+1)}\right] \mathbf{x}_{0}+\sum_{k=0}^{\infty} A^{k} \mathcal{L}^{-1}\left[s^{-(k+1) \alpha} B \mathbf{U}(s)\right]
\end{aligned}
$$

Berdasarkan Teorema 2.3, diperoleh

$$
=\sum_{k=0}^{\infty} \frac{A^{k} t^{k \alpha}}{\Gamma(k \alpha+1)} \mathbf{x}_{0}+\int_{0}^{t} \sum_{k=0}^{\infty} A^{k}(t-\tau)^{(k+1) \alpha-1} B \mathbf{u}(\tau) d \tau
$$

sehingga diperoleh

$$
\mathbf{x}(t)=\Phi_{0}(t) \mathbf{x}_{0}+\int_{0}^{t} \Phi(t-\tau) B \mathbf{u}(\tau) d \tau
$$

dimana

$$
\begin{aligned}
\Phi_{0}(t) & =\sum_{k=0}^{\infty} \frac{A^{k} t^{k \alpha}}{\Gamma(k \alpha+1)} \\
\Phi(t) & =\sum_{k=0}^{\infty} A^{k} \mathcal{L}^{-1}\left[s^{-(k+1) \alpha}\right] \\
& =\sum_{k=0}^{\infty} \frac{A^{k} t^{(k+1) \alpha-1}}{\Gamma(k+1) \alpha}
\end{aligned}
$$




\subsection{Sistem Linier Fractional Positif}

Perhatikan kembali sistem linier fractional (1.1).

Definisi 3.1. [6] Sistem fractional (1.1) dikatakan positif jika $\mathbf{x}(t) \in \mathbb{R}_{+}^{n}$ untuk setiap $\mathbf{x}_{0} \in \mathbb{R}_{+}^{n}$ dan semua input $\mathbf{u}(t) \in \mathbb{R}_{+}^{m}$, dengan $t \geq 0$

Lema 3.2. [6] Misalkan $A \in \mathbb{R}^{n \times n}, t \geq 0$ dan $0<\alpha<1$, maka

$\Phi_{0}(t)=\sum_{k=0}^{\infty} \frac{A^{k} t^{k \alpha}}{\Gamma(k \alpha+1)} \in \mathbb{R}_{+}^{n \times n}$, dan $\Phi(t)=\sum_{k=0}^{\infty} \frac{A^{k} t^{(k+1) \alpha-1}}{\Gamma[(k+1) \alpha]} \in \mathbb{R}_{+}^{n \times n}$

jika dan hanya jika $A$ adalah matriks Metzler.

\section{Bukti.}

$(\Rightarrow)$ Misalkan $A \in \mathbb{R}^{n \times n}$ dan persamaan (3.8) berlaku untuk $0<\alpha<1$, yaitu

$$
\begin{aligned}
& \Phi_{0}(t)=I_{n}+\frac{A t^{\alpha}}{\Gamma(\alpha+1)}+\frac{A^{2} t^{2 \alpha}}{\Gamma(2 \alpha+1)}+\cdots \in \mathbb{R}_{+}^{n \times n} \\
& \Phi(t)=I_{n} \frac{t^{\alpha-1}}{\Gamma(\alpha)}+A \frac{t^{2 \alpha-1}}{\Gamma(2 \alpha)}+A^{2} \frac{t^{3 \alpha-1}}{\Gamma(3 \alpha)}+\cdots \in \mathbb{R}_{+}^{n \times n}
\end{aligned}
$$

Karna $\Phi_{0}(t) \in \mathbb{R}_{+}^{n \times n}$ dan $\Phi(t) \in \mathbb{R}_{+}^{n \times n}$ untuk $t>0$ maka jelas bahwa $A$ adalah matriks Metzler.Begitupun sebaliknya untuk kasus $\Phi(\mathrm{t})$.

Teorema 3.3. [6] Sistem linier fractional (1.1) adalah positif jika dan hanya jika matriks $A$ adalah matriks Metzler dan $B \in \mathbb{R}_{+}^{n \times m}$

\section{Bukti.}

$(\Rightarrow)$ Perhatikan bahwa solusi sistem (1.1) diberikan oleh persamaan (3.5).

Misalkan sistem (1.1) adalah positif. Akan ditunjukkan bahwa $A$ adalah matriks Metzler dan $B \in \mathbb{R}_{+}^{n \times m}$. Karena sistem (1.1) adalah positif, maka berlaku $\mathbf{x}(t) \in \mathbb{R}_{+}^{n}$ untuk setiap $\mathbf{x}_{0} \in \mathbb{R}_{+}^{n}$ dan $t \geq 0$. Secara khusus, jika $\mathbf{u}(t)=0$ dan $\mathbf{x}_{0}=\mathbf{e}_{i}$, yaitu vektor kolom ke-i dari matriks Identitas maka (3.5) menjadi $\mathbf{x}(t)=\Phi_{0}(t) \mathbf{e}_{i}$. Akibatnya $\frac{d^{\alpha}}{d t^{\alpha}} \mathbf{x}(0)=A \mathbf{e}_{i}$. Karena trajektori dari sistem positif tidak mungkin meninggalkan orthant non negatif $\mathbb{R}_{+}^{n}$ maka $\frac{d^{\alpha}}{d t^{\alpha}} \mathbf{x}(0)=A \mathbf{e}_{i} \in \mathbb{R}_{+}^{n}$, dan mengakibatkan $A$ adalah matriks Metzler. Selanjutnya jika $\mathbf{x}_{0}=\mathbf{0}$ maka persamaan (3.5) menjadi $\frac{d^{\alpha}}{d t^{\alpha}} \mathbf{x}(0)=B \mathbf{u}(0) \in \mathbb{R}_{+}^{n} \cdot$ yang mengakibatkan $B \in \mathbb{R}_{+}^{n \times m}$.

$(\Leftarrow)$ Misalkan $A$ matriks Metzler dan $B \in \mathbb{R}_{+}^{n \times m}$. Akan ditunjukkan sistem (1.1) adalah positif. Karena $A$ adalah matriks Metzler, berdasarkan Lema (3.2) $\Phi_{0}(t) \in \mathbb{R}_{+}^{n}, \Phi(t) \in \mathbb{R}_{+}^{n}$ untuk $t \geq 0$, Akibatnya untuk setiap $\mathbf{x}_{0} \in \mathbb{R}_{+}^{n}$ dan $\mathbf{u} \in \mathbb{R}_{+}^{m}$ memberikan $\mathbf{x}(t) \in \mathbb{R}_{+}^{n}$, sehingga sistem (1.1) positif.

Sebagai contoh, perhatikan sistem (1.1) dengan

$$
A=\left[\begin{array}{ll}
0 & 1 \\
0 & 0
\end{array}\right] \in \mathbb{R}_{+}^{2 \times 2}, \quad B=\left[\begin{array}{l}
0 \\
1
\end{array}\right] \in \mathbb{R}_{+}^{2 \times 1},
$$


Jika diberikan $\mathbf{x}_{0}=\left[\begin{array}{l}1 \\ 1\end{array}\right] \in \mathbb{R}_{+}^{2 \times 1}$ dan $\mathrm{u}(\mathrm{t})=1$, maka solusi dari sistem linier tersebut dapat ditentukan sebagai berikut.

Dari persamaan (3.6) dan persamaan (3.7), diperoleh

$$
\begin{aligned}
& \Phi_{0}(t)=\sum_{k=0}^{\infty} \frac{A^{k} t^{k \alpha}}{\Gamma(k \alpha+1)}=I_{2}+\frac{A t^{\alpha}}{\Gamma(\alpha+1)}, \\
& \Phi(t)=\sum_{k=0}^{\infty} \frac{A^{k} t^{(k+1) \alpha-1}}{\Gamma[(k+1) \alpha]}=I_{2} \frac{t^{\alpha}-1}{\Gamma(\alpha)}+A \frac{t^{2 \alpha-1}}{\Gamma(2 \alpha)} .
\end{aligned}
$$

Karena

$$
A^{k}=\left[\begin{array}{ll}
0 & 0 \\
0 & 0
\end{array}\right], k=2,3,4,5, \cdots
$$

Selanjutnya subtitusikan persamaan (3.9) dan (3.10) dengan, $\mathrm{u}(\mathrm{t})=1(\mathrm{t})$ ke dalam persamaan (3.5), diperoleh

$$
\begin{aligned}
\mathbf{x}(t) & =\mathbf{x}_{0}+\frac{A \mathbf{x}_{0} t^{\alpha}}{\Gamma(\alpha+1)}+\int_{0}^{t}\left[\frac{B}{\Gamma(\alpha)}(t-\tau)^{\alpha-1}+\frac{A B}{\Gamma(2 \alpha)}(t-\tau)^{2 \alpha-1}\right] d \tau \\
& =\mathbf{x}_{0}+\frac{A \mathbf{x}_{0} t^{\alpha}}{\Gamma(\alpha+1)}+\frac{B t^{\alpha}}{\Gamma(\alpha+1)}+\frac{A B t^{2 \alpha}}{\Gamma(2 \alpha+1)} \\
& =\left[\begin{array}{l}
1 \\
1
\end{array}\right]+\left[\begin{array}{ll}
0 & 1 \\
0 & 0
\end{array}\right]\left[\begin{array}{l}
1 \\
1
\end{array}\right] \frac{t^{\alpha}}{\Gamma(\alpha+1)}+\left[\begin{array}{l}
0 \\
1
\end{array}\right] \frac{t^{\alpha}}{\Gamma(\alpha+1)}+\left[\begin{array}{ll}
0 & 1 \\
0 & 0
\end{array}\right]\left[\begin{array}{l}
0 \\
1
\end{array}\right] \frac{t^{2 \alpha}}{\Gamma(2 \alpha+1)} \\
& =\left[\begin{array}{l}
1 \\
1
\end{array}\right]+\left[\begin{array}{l}
1 \\
0
\end{array}\right] \frac{t^{\alpha}}{\Gamma(\alpha+1)}+\left[\begin{array}{l}
0 \\
1
\end{array}\right] \frac{t^{\alpha}}{\Gamma(\alpha+1)}+\left[\begin{array}{l}
1 \\
0
\end{array}\right] \frac{t^{2 \alpha}}{\Gamma(2 \alpha+1)} \\
& =\left[\begin{array}{c}
1+\frac{t^{\alpha}}{\Gamma(\alpha+1)}+\frac{t^{2 \alpha}}{\Gamma(2 \alpha+1)} \\
1+\frac{t^{\alpha}}{\Gamma(\alpha+1)}
\end{array}\right] \in \mathbb{R}_{+}^{2 \times 1}
\end{aligned}
$$

Untuk $\alpha=0.5$, diperoleh solusi sistem (1.1) adalah

$$
\mathbf{x}(t)=\left[\begin{array}{c}
1+2 \sqrt{\frac{t}{\Pi}}+t \\
1+2 \sqrt{\frac{t}{\Pi}}
\end{array}\right]
$$

\section{Kesimpulan}

Solusi sistem persamaan diferensial fractional linier (1.1) adalah

$$
\mathbf{x}(t)=\Phi_{0}(t) \mathbf{x}_{0}+\int_{0}^{t} \Phi(t-\tau) B \mathbf{u}(\tau) d \tau
$$


dimana

$$
\begin{aligned}
& \Phi_{0}(t)=\sum_{k=0}^{\infty} \frac{A^{k} t^{k \alpha}}{\Gamma(k \alpha+1)} \\
& \Phi(t)=\sum_{k=0}^{\infty} \frac{A^{k} t^{(k+1) \alpha-1}}{\Gamma(k+1) \alpha}
\end{aligned}
$$

Sistem persamaan diferensial fractional linier (1.1) adalah positif jika dan hanya jika matriks $A$ adalah matriks Metzler dan $B \in \mathbb{R}_{+}^{n \times m}$.

\section{Ucapan Terima kasih}

Penulis mengucapkan terima kasih kepada bapak Dr. Mahdhivan Syafwan, ibu Hazmira Yozza, M.Si, bapak Dr. Ahmad Iqbal Baqi selaku dosen penguji yang telah memberikan masukan dan saran sehingga makalah ini dapat diselesaikan dengan baik.

\section{Daftar Pustaka}

[1] Anton, H. 2004. ALjabar Linear Elementer . Edisi Kedelapan. Terjemahan Bahasa Indonesia. Erlangga: Jakarta

[2] Caputo, M. 1967. Linier Model of Dissipation Whose $Q$ is Almost Frequency Independent-II. Geophysical Jurnal International, 13(5): 529 - 539

[3] Duan, G.R. Analysis and Design of Descriptor Linear Systems. Springer, New York.

[4] Hendriks, E.J. and P.H. Sorensen. 2008 Linier Systems Control. Springer-Verlag. Berlin Heidenberg

[5] Kaczorek, T. 2008. Fractional Positive Continuous-Time Linear Systems and Their Reachability. Int. J. Appl. Math. Comput. Sci, 18(2): 223 - 228

[6] Kaczorek, T. 2011 . Selected Problem of Fractional Systems Theory. SpringerVerlag Berlin Heidelberg

[7] Mitkowski. W. 2018. Dynamical Properties of Metzler Systems. Bulletin of The Polish Academy of Sciences Technical Sciences. 56(4): 309 - 312 